Musées, Patrimoine et Culture scientifiques et techniques

$171 \mid 2017$

mai-juin 2017

\title{
Animer une communauté locale de culture scientifique et technique grâce au web social
}

\section{Marion Sabourdy}

\section{OpenEdition \\ Journals}

Édition électronique

URL : http://journals.openedition.org/ocim/1778

DOI : $10.4000 /$ ocim. 1778

ISSN : 2108-646X

Éditeur

OCIM

Édition imprimée

Date de publication : 1 mai 2017

Pagination : 22-28

ISSN : 0994-1908

Référence électronique

Marion Sabourdy, "Animer une communauté locale de culture scientifique et technique grâce au web social », La Lettre de I'OCIM [En ligne], 171 | 2017, mis en ligne le 01 mai 2018, consulté le 22 avril 2019. URL : http://journals.openedition.org/ocim/1778 ; DOI : 10.4000/ocim.1778

Ce document a été généré automatiquement le 22 avril 2019

Tous droits réservés 


\section{Animer une communauté locale de culture scientifique et technique grâce au web social}

Marion Sabourdy 
Le Forum des projets d'Echosciences Grenoble s'est tenu tous les ans, de 2012 à 2016 et a rassemblé à chaque édition entre 50 et 90 acteurs de CSTI du territoire grenoblois. L'occasion pour les membres de la plateforme de se retrouver et d'échanger.
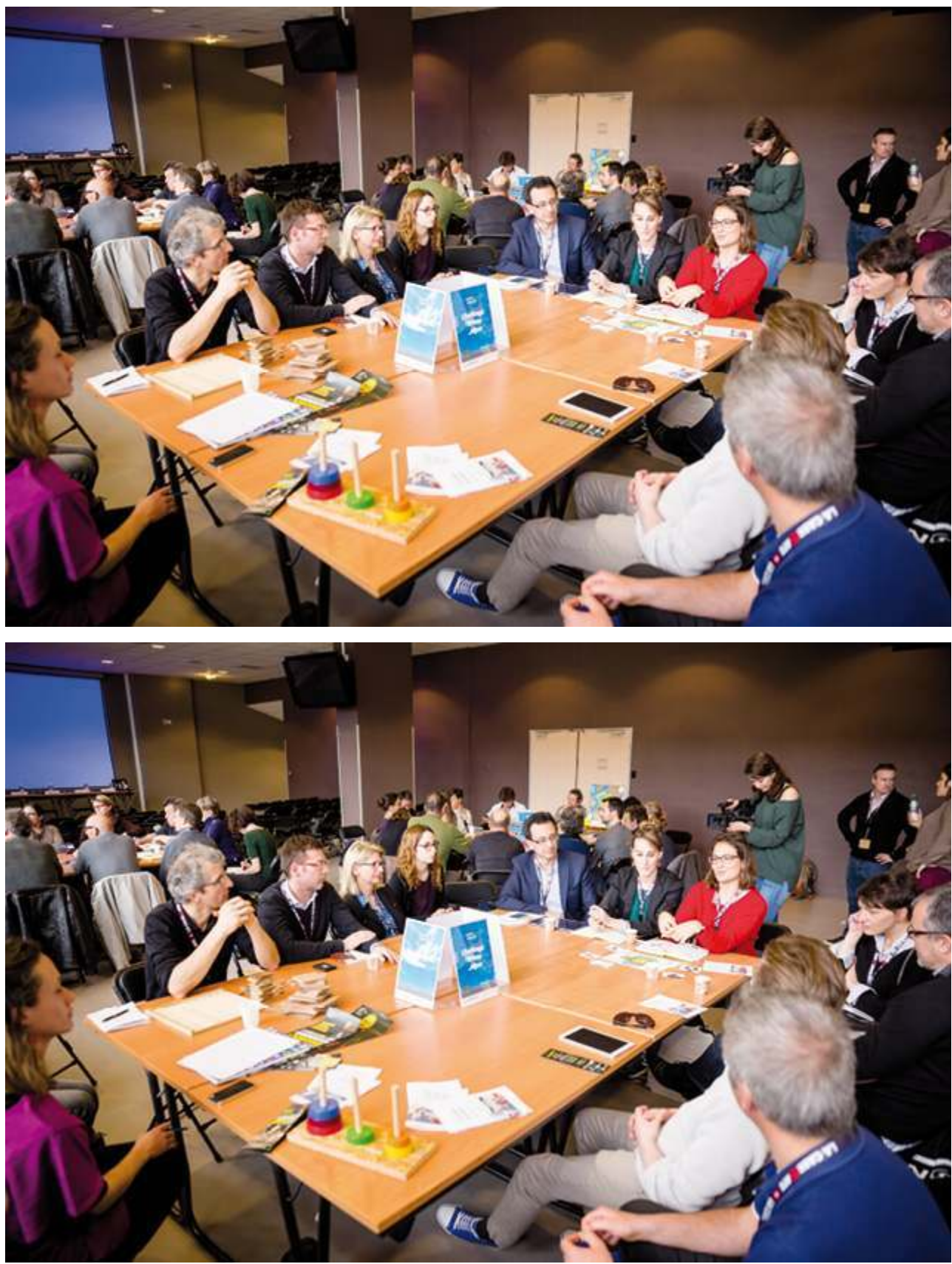

(c) UtopikPhoto pour la Casemate

1 Premier centre de sciences français, coordinateur de la Fête de la science depuis ses débuts il y a 26 ans à l'échelle régionale puis départementale, éditeur du journal papier À découvrir pendant une dizaine d'années sur l'actualité de la culture scientifique, technique et industrielle (CSTI) en Rhône-Alpes, La Casemate, le Centre de culture scientifique, technique et industrielle (CCSTI) de Grenoble, a toujours été au contact des acteurs de son territoire et soucieux de les mettre en lien, de travailler avec eux et de les valoriser.

2 Mais jusqu'au début des années 2010, aucune initiative de cartographie publique du territoire métropolitain en matière de CSTI n'avait encore été entreprise. Dès le début de 
l'année 2011, La Casemate initiait alors une série d'ateliers thématiques, largement ouverts, afin de concevoir collectivement de nouvelles formes et pratiques de rencontres et de collaborations à l'échelle du territoire, avec un soutien fort des collectivités territoriales (Grenoble Alpes Métropole notamment) et des Investissements d'Avenir ${ }^{1}$.

Laurent Chicoineau, directeur de la Casemate, résumait ainsi les grands enjeux : "Mieux faire connaître toutes les actions menées sur l'agglomération pour partager et diffuser les connaissances scientifiques. Rapprocher tous ceux qui s'impliquent dans ces activités. Mobiliser encore plus d'habitants sur les questions d'innovation, de création, de développement durable et de recherche. Avoir une vision d'ensemble, renforcer le maillage et la mutualisation sur un territoire sans casser les dynamiques existantes - au contraire, en renforçant les capacités d'action de chacun"2.

Aperçu d'une séance de créativité avec les partenaires du projet, en novembre 2011, qui mènera au nom d'Echosciences.

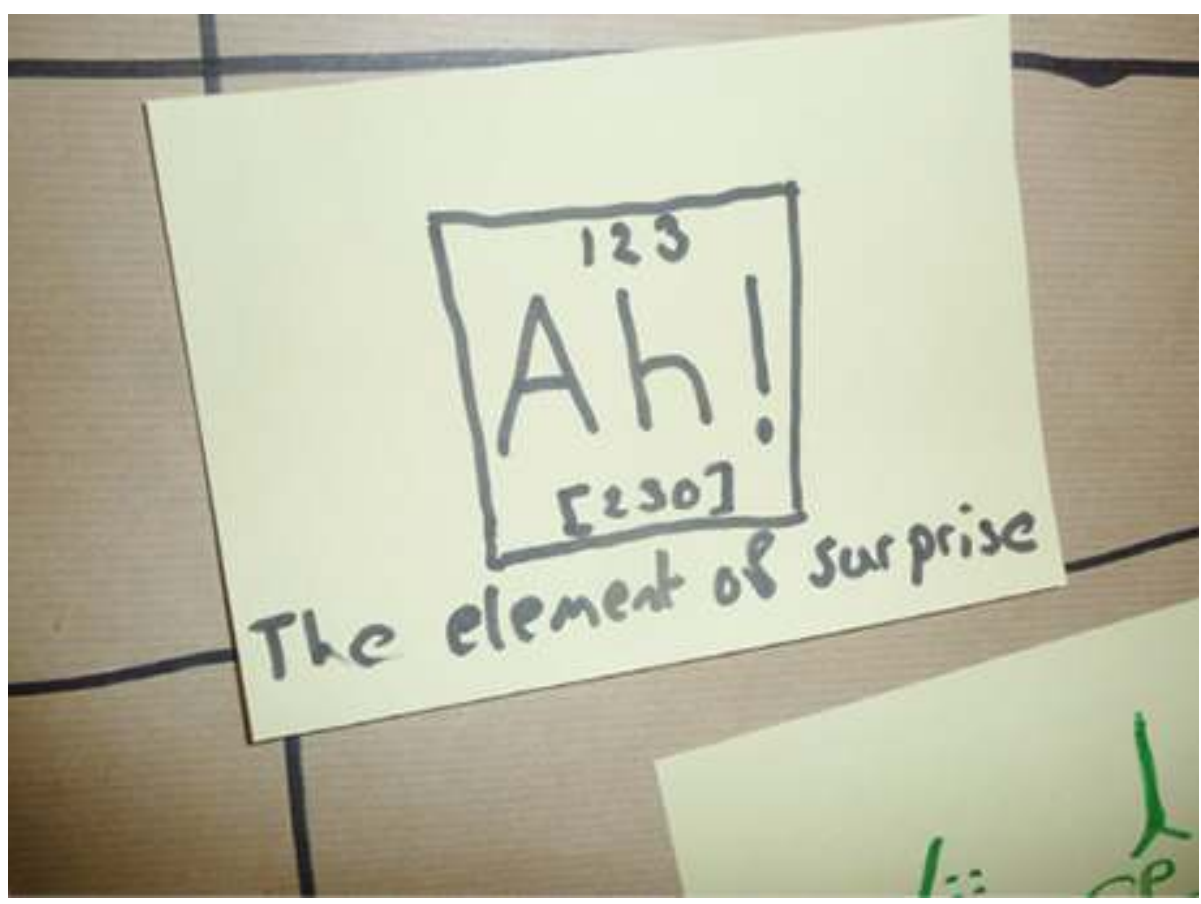

(C) La Casemate

4 Parmi les nouvelles formes de collaboration à disposition, le recours aux outils numériques et au web social a rapidement été abordé ${ }^{3}$. En effet, depuis une dizaine d'années, les réseaux sociaux numériques ont fait évoluer les métiers de la CSTI, bousculant leur relation aux publics. Ils ont également permis à de tous nouveaux acteurs d'émerger, à la faveur, entre autres, de plateformes de blogging ${ }^{4}$, de micro-blogging (Twitter) ou encore de partage de vidéos (Youtube notamment).

5 En février 2012, au terme d'une année de discussions et de créativité collective, un outil naît de cette concertation des parties prenantes. Un outil qui va bien au-delà d'un simple annuaire et intègre les dimensions participatives du web. Cet outil, c'est Echosciences, dont le nom, lui aussi imaginé lors d'une de ces séances de "brainstorming", englobe à la fois la communauté d'acteurs et la plateforme en tant que telle. Il s'agit du premier réseau social dédié aux acteurs de CSTI d'un territoire. 


\section{Echosciences, en bref}

6 Echosciences n'est pas un site de vulgarisation scientifique, ni un magazine de publication scientifique. C'est un outil de veille culturelle participative : il permet un recensement contributif des initiatives, événements, bonnes pratiques, actualités de la CSTI locale, fait par et pour les amateurs et professionnels de la CSTI $^{5}$. C'est aussi un outil d'expression où des personnes et des institutions donnent à voir ce qu'elles font. Et, c'est enfin une plateforme de mise en contact, qui permet de découvrir et d'échanger.

7 Avec cet outil, nous proposons une nouvelle approche, basée sur la mise en réseau, la veille collective et la montée en compétence des amateurs et professionnels de la CSTI d'un territoire. Cette approche est basée sur la confiance, l'horizontalité et la réflexivité. Cela représente un réel "pas de côté" par rapport aux approches traditionnelles de la CSTI, relativement "descendantes". Nous souhaitons proposer aux acteurs des outils qui leur permettent de se raconter, de valoriser leurs actions et d'en découvrir d'autres.

Echosciences peut également être vu comme un "bac à sable" pour tester des usages, réfléchir sur sa pratique, présenter les coulisses d'un projet ou d'un événement, parler de ses échecs aussi. Le format et l'accompagnement incitent le contributeur à tirer parti de l'outil, à s'en inspirer voire à le détourner.

9 Le pari de cette plateforme - et, nous le pensons, ce qui fait sa réussite - est de s'adresser à une communauté d'intérêt et de casser l'approche traditionnelle, par institution et par expertise. Echosciences défend une vision moderne de la CSTI - horizontale, participative, dynamique - et des liens entre culture scientifique et culture numérique. En résumé, Echosciences tend à représenter au mieux les personnes qui aiment, parlent et pratiquent les sciences sur un territoire et à leur donner les outils pour se mettre en contact.

Voilà pourquoi nous avons souhaité ne pas mettre de "barrière" à l'entrée : les inscriptions se font automatiquement, sans validation de la part de l'administrateur. Quant à la modération, elle est faite a posteriori, c'est-à-dire après publication, par la communauté, via un bouton de signalement. Une telle approche ne minimise pas les risques inhérents au web. Nous sommes d'ailleurs particulièrement attentifs aux questions de modération et de propriété intellectuelle. Mais c'est le risque minimal à prendre pour pouvoir jeter réellement les bases d'un travail collectif.

11 Enfin, Echosciences n'a pas pour but de se substituer à la communication institutionnelle de chacun de ses membres ou à la production de contenus et outils de vulgarisation et médiation. Au contraire, il les accompagne et les facilite en rassemblant en un même endroit les acteurs, tout en créant des liens avec leurs sites ou leurs ressources. Par exemple, la rubrique "Ressources" de la communauté Atout Cerveau d'Echosciences Grenoble propose, sous format PDF, des mini-livres sur le cerveau, des cartes postales sur l'étude du stress... Une partie de ces ressources a été utilisée par des acteurs à Montpellier... qui ont ensuite documenté leurs actions sur Echosciences Occitanie.

\section{Qui sont les membres de la communauté ?}

12 À qui s'adresse-t-on ? Qu'entendons-nous par "acteurs de CSTI" ? La particularité des réseaux sociaux numériques - Echosciences n'y fait pas exception - est de permettre au public de s'exprimer. La plateforme s'adresse donc à tout habitant d'un territoire 
intéressé par les sciences et techniques, à partir du lycée, et sans distinction d'âge, de niveau d'étude ou de métier. Concrètement, on retrouve des médiateurs, des enseignants, des élèves, étudiants et doctorants, des scientifiques (ingénieurs, chercheurs, enseignants-chercheurs...), des représentants d'institutions scientifiques ou culturelles (instituts, laboratoires, musées, associations, universités...), des représentants de collectivités locales ainsi que des amateurs de sciences ou des citoyens curieux. Au-delà de ces acteurs, Echosciences s'adresse également à ceux qui peuvent parler de sciences occasionnellement : blogueurs, vidéastes, photographes, illustrateurs, auteurs, professionnels du livre, enseignants de matières "non scientifiques"...

Origine géographique des lecteurs d'Echosciences Grenoble en France.

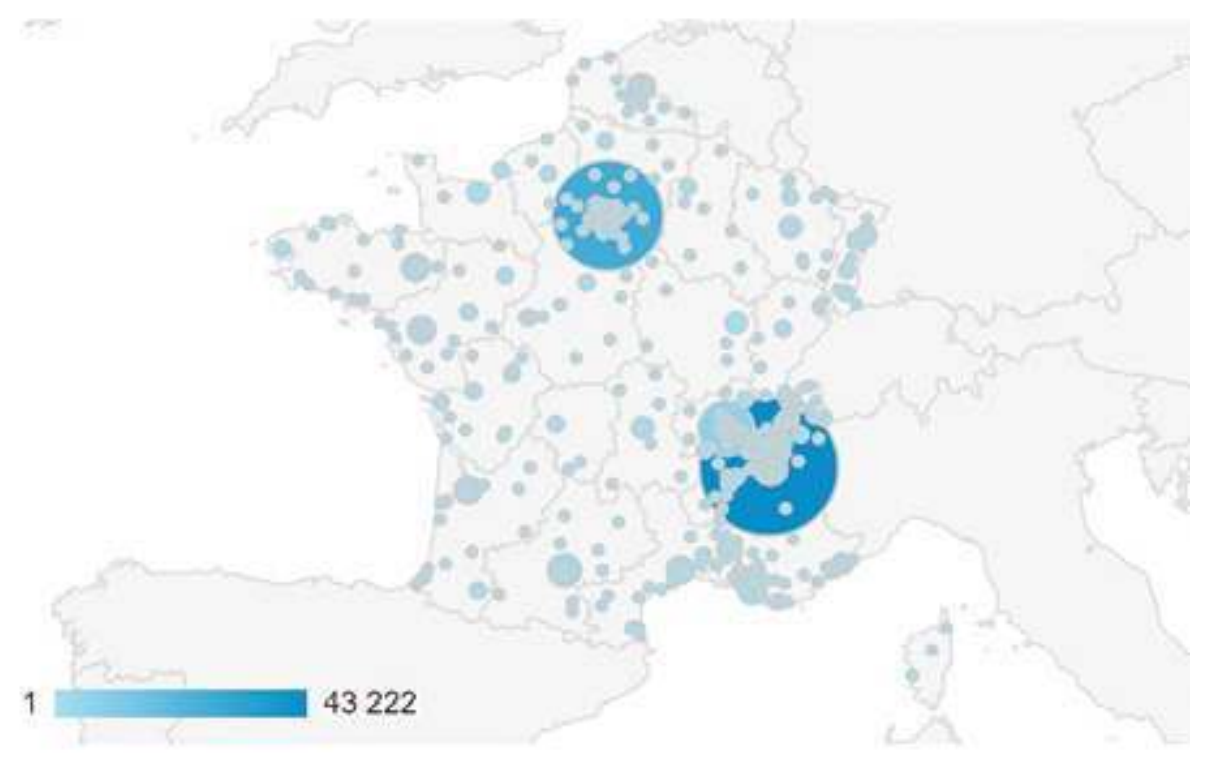

(c) Google Analytics

En 2016, Echosciences Grenoble a compté 129400 visiteurs uniques et 381000 pages vues soit une moyenne de 10800 visiteurs et 32000 pages vues par mois ou encore 350 visiteurs et 1000 pages vues par jour. $27 \%$ des visiteurs ont entre 25 et 34 ans, $24 \%$ entre 35 et 44 ans et $16 \%$ entre 18 et 24 ans. La parité est respectée. Parmi les visiteurs français, $42 \%$ proviennent de l'ancienne région Rhône-Alpes (dont 2/3 de la ville de Grenoble) et $24 \%$ d'Ile-de-France. $30 \%$ de la totalité des lecteurs consultent le site en mobilité (smartphone ou tablette). Du côté des contributeurs, on compte 147 femmes, 136 hommes et 60 "neutres" (comptes institutionnels) soit un total de 343 contributeurs, qui ont posté près de 2000 contributions depuis la création du site. Parmi eux, $77 \%$ ont publié entre 1 et 5 contributions sur Echosciences ${ }^{6}$.

\section{La Casemate, entre culture scientifique et culture numérique}

À Grenoble, la Casemate a depuis les années 1970 mis en scène l'informatique puis le numérique dans ses expositions et offre depuis quelques années un terreau fertile pour les expérimentations à la frontière entre CSTI et numérique. Notamment les expositions Informatique et vie quotidienne en 1978, L'Homme communiquant en 1997, Chroniques en TIC en 2002, Tous Connectés en 2011, les événements "Quais des clics" en 1999, "Grenoble web session" en 2002 et 2003, la "Nuit du retrogaming" en 2011, 
l'édition de l'application de la Fête de la science en 2011, la participation à Museomix en 2013 et à la Museum Week en 2015 et 2016, les trois Scientific Game Jam entre 2015 et 2017, entre autres.

\section{La genèse d'un projet collaboratif} l'activité de CSTI de leur territoire. Cet outil leur facilite également le travail : identification, cartographie et valorisation des structures, dialogue et contact privilégié avec les acteurs (dont certains parfois éloignés de la CSTI comme des musées d'art), données chiffrées concernant leur réseau, liens et retours d'expérience avec les animateurs des autres Echosciences, participation des publics à la conception de projets...

Enfin, cette dynamique a pour but de faire progresser ensemble tous les acteurs dans leurs métiers de médiation et de vulgarisation scientifique, en s'inspirant les uns des autres et en s'acculturant au numérique. Laurent Chicoineau le confirme : "Avec ce projet, nous avons radicalement transformé les relations que nous entretenions avec les autres acteurs de CSTI du territoire. De tête de réseau, avec toutes les connotations centralisatrices et hiérarchiques que ce terme revêt en France, nous sommes passés à facilitateurs de la mise en réseau, du partage et de la mutualisation entre acteurs, sans porter aucun jugement sur les contenus et offres apportés par les uns et les autres. Nous avons créé une nouvelle grande communauté à l'échelle de notre territoire métropolitain, motivée par le partage des savoirs et des innovations, fondée sur la confiance, la bienveillance et la solidarité - valeurs indispensables pour défendre et renforcer nos activités culturelles et éducatives dans un contexte d'accès aux ressources de plus en plus tendu". 
Exemple de lien entre une plateforme web et un événement physique comme la Fête de la science : le programme papier renvoie vers des contenus en ligne (portraits, agenda).

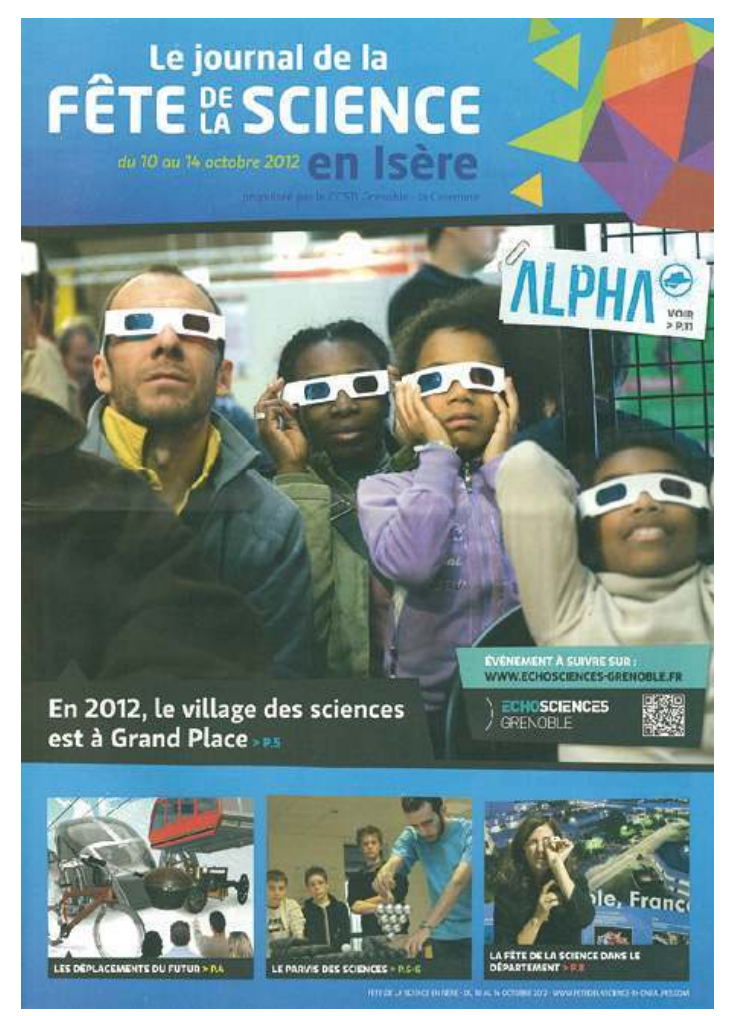

(c) Studio BamBam pour La Casemate

\section{Les différents rôles, du lecteur à l'administrateur} n'ont pas toutes le même degré d'investissement. On distingue notamment les "lecteurs" (ou "visiteurs"), qui consultent simplement le site, une seule fois ou de manière plus récurrente. Parmi eux, les plus investis peuvent aller jusqu'à commenter un contenu ${ }^{8}$.

Les personnes qui font la démarche de s'inscrire sur Echosciences deviennent des "membres". Ils peuvent alors suivre d'autres membres (et être avertis de leurs publications), suivre des tags (et être avertis lorsque ceux-ci sont utilisés) et publier des articles, événements, annonces ou dossiers. Chaque semaine, ils reçoivent dans leur boîte mél, s'ils le souhaitent, un résumé des activités de la plateforme. Un membre qui a publié au moins un contenu sur la plateforme est alors qualifié de "contributeur" (dans les faits, il a les mêmes droits qu'un membre mais a franchi un palier supplémentaire d'engagement). Ainsi, début mars 2017, le nombre total de membres (i.e. personnes inscrites) sur les 6 Echosciences en ligne était de 1750 . Parmi eux, les contributeurs de ces plateformes avaient alors publié 1500 articles, 1630 événements, 200 annonces, 85 dossiers et 75 projets.

Au-dessus des contributeurs, on retrouve les administrateurs de communauté. Ces personnes se sont vues attribuer des droits d'administration sur une ou plusieurs communauté(s). Ils peuvent donc éditer celles-ci et y ajouter des contributeurs ou d'autres administrateurs. Au-delà de cette simple appellation, ces personnes ont à la fois 
un rôle de leader auprès des membres de leur propre communauté et un rôle d'ambassadeur auprès de l'administrateur d'Echosciences. Ils proposent des contenus, recrutent de nouveaux contributeurs, échangent avec l'animateur du site, qui s'appuie sur eux pour faciliter son travail...

21 Enfin, chaque plateforme Echosciences est animée par un nombre très restreint d'administrateurs (généralement entre un et trois), qui disposent de droits sur la personnalisation graphique de la plateforme ainsi que sur l'édition des contenus. Au-delà de ces prérogatives techniques ou esthétiques (correction de fautes, ajout de liens, recherche iconographique si nécessaire), le véritable rôle de l'animateur ou l'animatrice d'Echosciences est de faire... que la communauté vive toute seule. Pour cela, sa posture est celle d'un facilitateur et son rôle est d'accompagner les contributeurs, de les soutenir et d'amener autant que possible chaque personne à s'engager un peu plus. Au besoin, l'animateur peut former les membres de la communauté à des notions d'écriture, de veille, de recherche iconographique ou d'animation de communauté. L'objectif étant, à terme, de permettre "l'empowerment" (ou montée en compétences) de tous les membres de la communauté sur les questions de culture numérique en général. À Grenoble, cela a été le cas avec le Muséum, qui a notamment lancé la plateforme participative Nature Isère 9

22 Au quotidien, a minima, son travail consiste à valoriser les productions des membres et inviter d'autres personnes à rejoindre la communauté. Dans les faits et selon les ressources humaines consacrées à l'animation de cette plateforme (généralement, entre $1 / 2$ et un temps plein), les administrateurs peuvent solliciter des retours des lecteurs (via des enquêtes) et de la communauté (notamment les propositions d'amélioration de la plateforme), représenter la communauté et valoriser les contenus produits par les membres auprès d'autres acteurs du territoire (et au-delà), engager des discussions sur des thématiques transverses, au besoin accompagner le montage de certains projets, voire des campagnes de financement participatif... 
Contributeurs et administratrices de la communauté Atout Cerveau, sur Echosciences Grenoble. Le bouton "suivre" permet à un membre d'être alerté lorsque l'un d'eux publie un contenu.

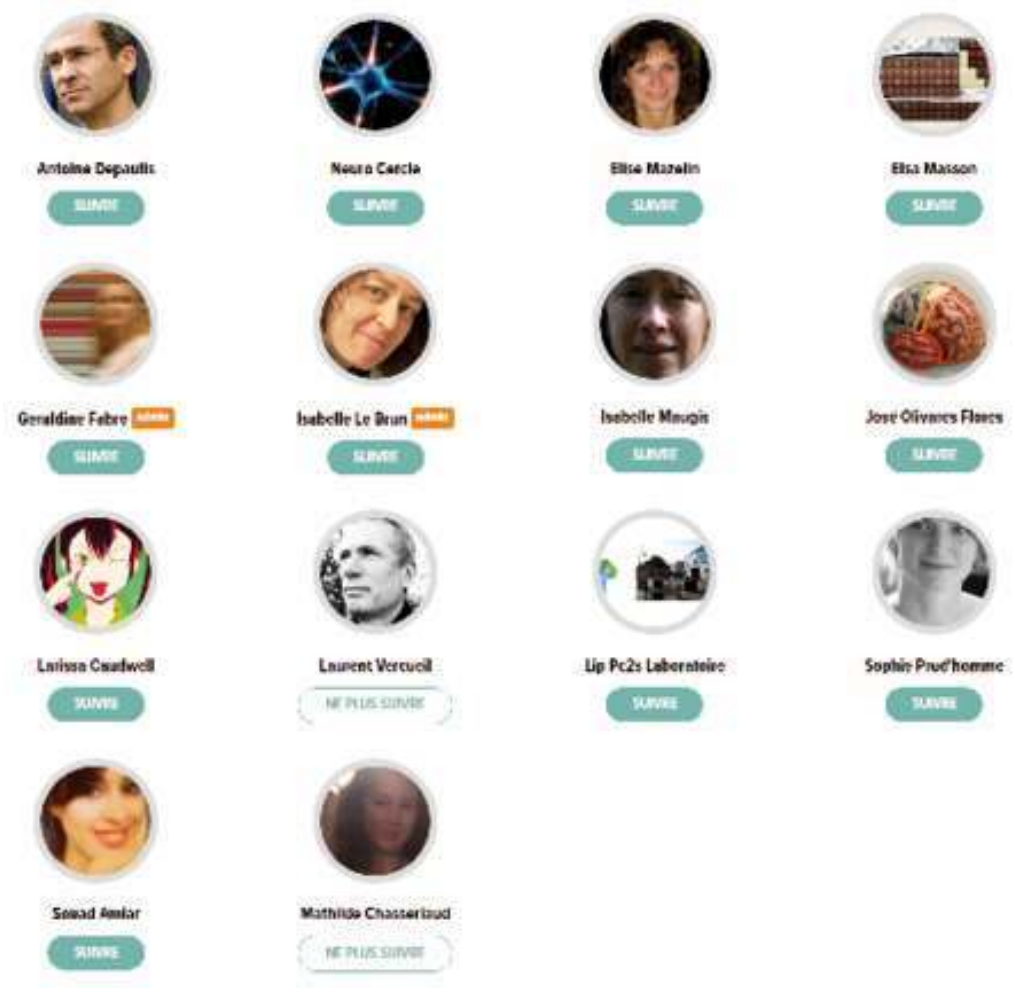

(c) Echosciences

\section{Aucun Echosciences n'est une île}

L'animation d'une plateforme Echosciences s'accompagne d'une dynamique de veille et de promotion de la communauté. Ces dernières peuvent se tenir sur des réseaux sociaux généralistes (Twitter, Facebook...) mais aussi, selon le temps dont dispose l'animateur, sur les plateformes de partage de photographies (Flickr, Instagram...) de vidéos (Youtube, Dailymotion, Vimeo...) ou encore de crowdfunding (KissKissBankBank, Ulule, Kickstarter...).

Le flux de Twitter et la page Facebook de la communauté sont d'ailleurs présentés dès la page d'accueil, via des widgets. La maquette de la plateforme fait la part belle aux photographies, GIF (format d'images animées) et autres vidéos et les campagnes de financement participatif ont même une rubrique dédiée.

Une telle présence sur le web permet de repérer des contenus produits par des acteurs de CSTI hors Echosciences et de les faire entrer dans la communauté. C'est ainsi que nous avons pu valoriser des contenus produits par des blogueurs locaux (par exemples des idées de sorties culturelles rédigées par une maman blogueuse), rencontrer et faire le portrait d'illustrateurs naturalistes et de photographes indépendants et approcher les acteurs des nouvelles formes de médiation comme des vidéastes, les animateurs du compte @EnDirectDuLabo, les personnes qui organisent et live-tweetent l'événement "Ma Thèse en 180 secondes"... 

de chaque plateforme de pouvoir interagir avec les membres et les contenus des autres plateformes (suivre une personne, ajouter un contenu dans un dossier, s'abonner à un tag...). Par ailleurs, une API ("Application Programming Interface" ou interface de programmation $)^{10}$ a été développée en vue de permettre à des sites tiers de récupérer et d'intégrer les articles et les événements en provenance des différents Echosciences.

Tweet de valorisation des événements de CSTI qui se tiennent en Hauts-de-France, par la plateforme Echosciences de cette région.
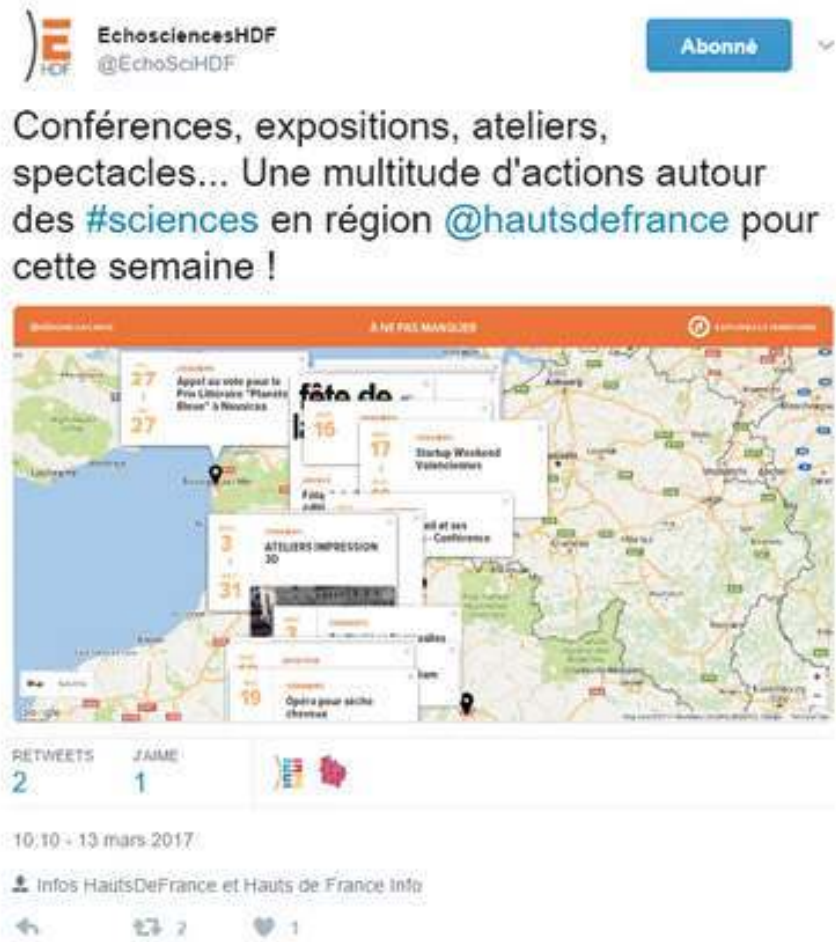

(c) Echosciences Hauts-de-France 
Toute structure ou entité dont le rôle est d'animer l'activité de CSTI de son territoire peut ainsi tirer parti d'Echosciences pour mener à bien sa mission. Dans ce cadre, un site vitrine a été conçu pour présenter le réseau (Echosciences.com). Une page Facebook et un compte Twitter (@EchoSciTeam) sont animés afin de valoriser les différents Echosciences et leurs membres.

31 Les animateurs des différents Echosciences échangent régulièrement au sujet de l'animation de leurs territoires respectifs, des différentes formes de médiation numérique ainsi que sur les améliorations techniques possibles de la plateforme. Ces échanges font monter en compétences tous les membres du groupe et profitent à terme à l'ensemble des acteurs. À l'écoute de leur réseau, les animateurs font remonter des informations concrètes qui permettent une constante mise à jour de la plateforme, ce qui bénéficie à tous.

Zones géographiques couvertes par les plateformes Echosciences en ligne en mars 2017 (en couleurs) et celles qui le seront au courant de l'année (en gris).

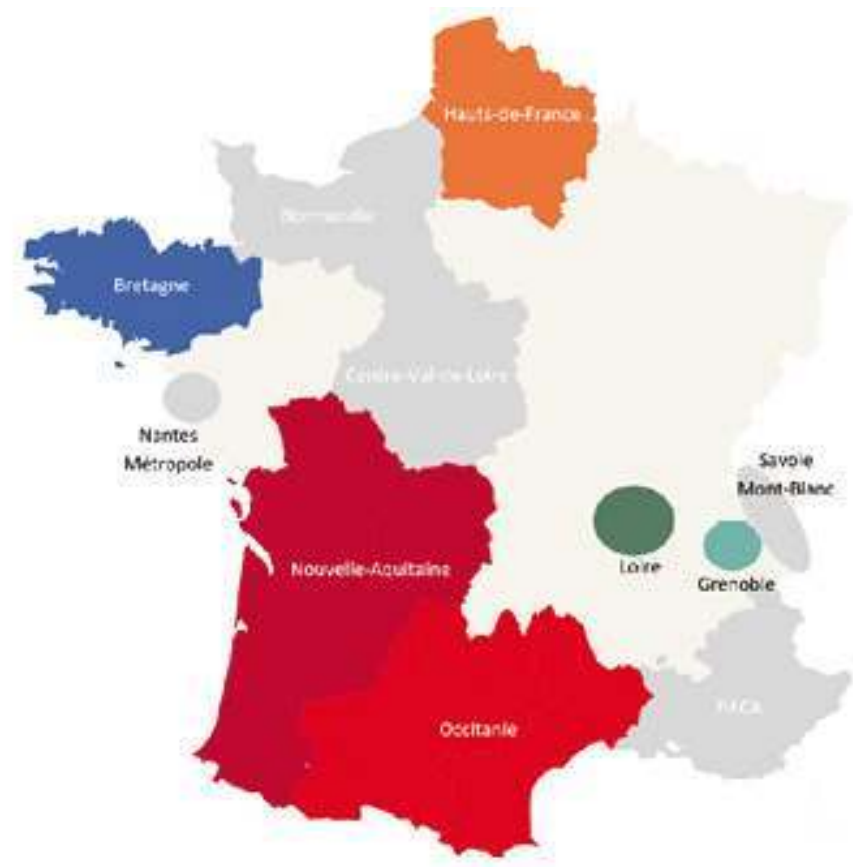

(c) Fond de carte comersis.com

\section{Conclusion}

Echosciences est une initiative unique en son genre. Née à Grenoble, à la faveur d'une réflexion sur les acteurs de la CSTI de ce territoire, cette plateforme est devenue, petit à petit, un point d'accès incontournable aux acteurs et initiatives de CSTI dans plusieurs territoires. Ce déploiement de la plateforme pose la question de la gouvernance et du modèle économique du dispositif.

Le coût de développement de la version actuelle d'Echosciences est de $94000 €$ pour la plateforme et $40000 €$ pour "le cloud". Echosciences a été financé par les Investissements d'Avenir (via le programme Inmédiats), ainsi que par la Ville de Grenoble et Grenoble Alpes Métropole. Le déploiement d'une plateforme sur un nouveau territoire coûte, quant 
à lui, moins de $3000 €$ la première année (installation et hébergement) puis environ 1200 $€$ par an (hébergement).

Nous souhaitons associer les animateurs des différents Echosciences dans une organisation propre, de type coopérative, indépendante de la Casemate. L'objectif étant de participer à une dynamique collective, à l'échelle nationale, de valorisation et de structuration de la CSTI à partir de nos territoires. Il nous apparaît certain que la pérennité des plateformes sera assurée si chaque territoire est associé à toutes les décisions la concernant. Les premières rencontres physiques entre animateurs et avec les acteurs intéressés par cette démarche auront lieu à Bordeaux du 5 au 7 juillet 2017 lors du congrès de l'Amcsti.

Le texte suivant figure en encadré dans l'édition papier :

\section{Que trouve-t-on sur Echosciences?}

\section{Des cartes}

Elles permettent de découvrir et de documenter collectivement le territoire. On les retrouve en page d'accueil pour épingler les événements et articles les plus intéressants, dans l'agenda pour mettre en avant les prochains événements et dans la rubrique "À visiter" pour présenter tous les lieux culturels scientifiques du territoire.

\section{Des espaces personnels}

Un espace "Profil" qui constitue l'identité des membres sur le réseau et un espace privé "Mon Echosciences", sorte de tableau de bord qui rassemble toutes les actions d'un membre, ses publications, ses notifications et lui propose un flux d'information personnalisé.

\section{Différentes formes de contribution}

Dans la rubrique "Articles", les membres postent des retours d'expérience, des reportages, des portraits, des débats (nous nous sommes notamment inspirés du site de débats publiques québécois Escarmouches www.escarmouches.ca)... Les rubriques "Agenda", "Annonces" et "Projets" permettent de relayer respectivement un événement (atelier, conférence, exposition...), une annonce (offre d'emploi, appel à participation, concours...) ou une campagne de financement participatif. Quant à la rubrique "Dossier", elle permet d'agréger des contenus et ainsi proposer un travail de "curation de contenus" directement inspiré du web (la curation de contenu est une pratique qui consiste à sélectionner, éditer et partager les contenus les plus pertinents du web sur un sujet donné pour leur offrir une plus grande visibilité).

\section{Des communautés}

Il s'agit de sous-ensembles de la plateforme permettant à de petites communautés d'intérêt ou de projet de se structurer. Il peut s'agir de communautés thématiques (neurosciences, robotique...), de groupes de chercheurs et communicants qui se rassemblent à la faveur d'un événement (Années internationales, Fête de la science, 
Semaine du cerveau...), de structures ne disposant pas de site internet... Chaque communauté administre son espace et peut le personnaliser et l'animer en autonomie.

\section{Une navigation par tags}

Toutes les publications postées dans ces différentes rubriques ont un point commun : elles sont toutes qualifiées avec des tags (mots-clés), qui permettent une navigation thématique. Ces tags sont choisis librement par les contributeurs. N'importe quel membre peut s'abonner à un tag et recevoir une alerte mail à chaque fois que celui-ci est utilisé. Ce choix de baser la navigation sur les tags choisis librement s'est fait en 2015, au moment de la refonte complète de la plateforme. Ce mode de fonctionnement, même s'il a ses limites (notamment les fautes d'orthographe, que l'animateur peut corriger), permet de dessiner un paysage sémantique bien plus fin, de la CSTI d'un territoire et pourrait mener à un projet de recherches en sciences humaines autour de ces données.

Carte des lieux de CSTI à visiter en Isère. Chaque épingle sur la carte renvoie à une page décrivant les structures.

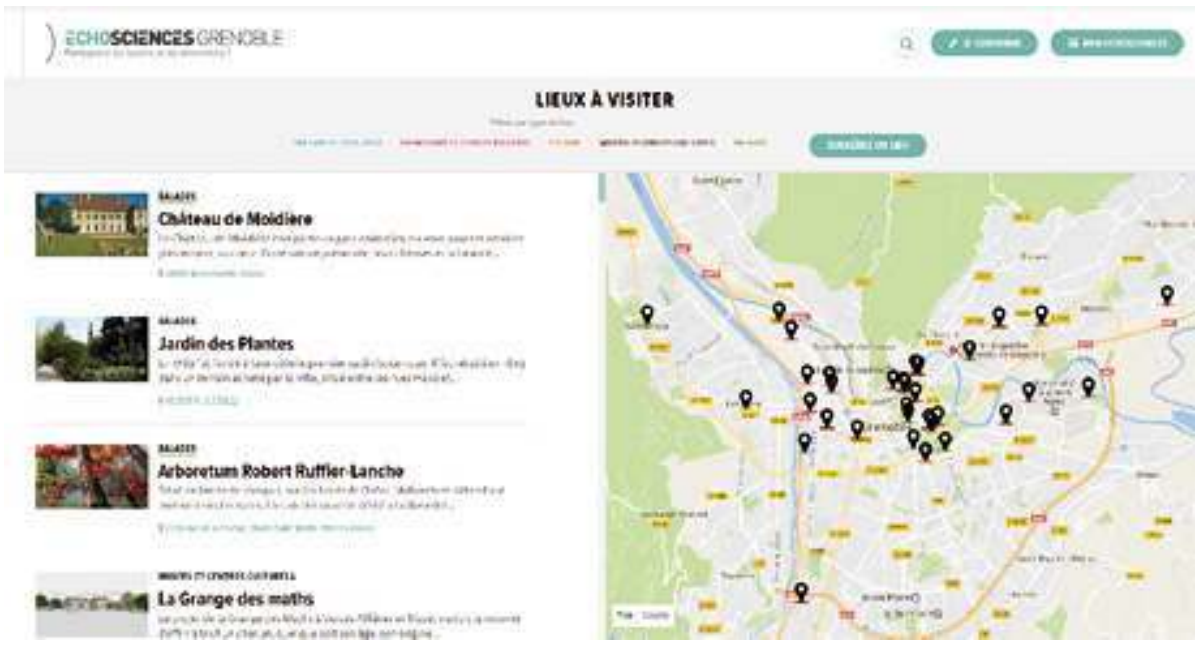

(c) Echosciences 
Exemple d'une page "Profil".

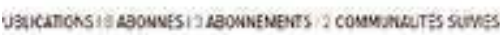

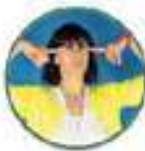

Q STIE WEB $Y$ TWITE I in LWES

\section{Corinne Poinas}

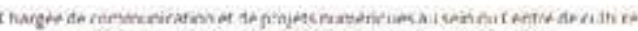

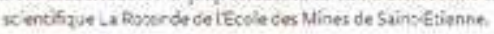

\section{kenus siak}

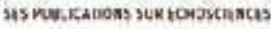

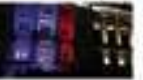

M>een

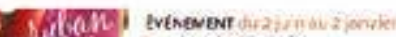

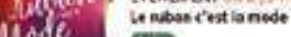

Uate

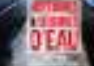

$\rightarrow$

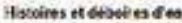

(c)

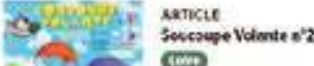

cincs

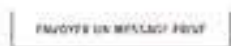

-

son actwite sas schosciescss

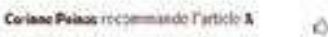

andervesse in temes smater

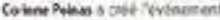

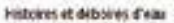

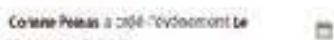

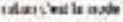

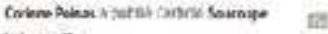

mintenc?

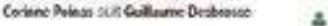

(c) Echosciences

Quatre exemples de communautés consultables sur Echosciences Grenoble.

\section{LES COMMUNAUTÉS}
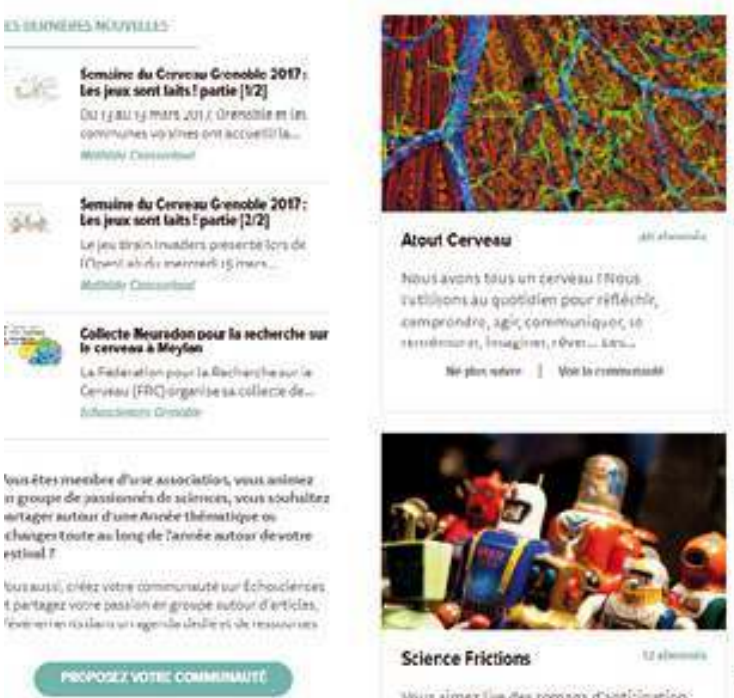

\section{Atout Cervesu}

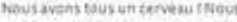

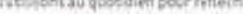

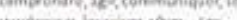

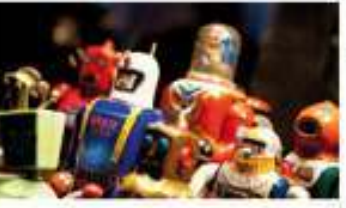

Stience Frictions

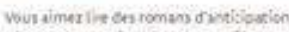

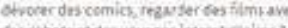
Gebrebots

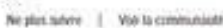

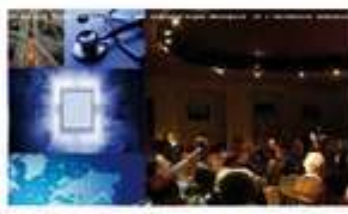

Cafés scienses et Citopens ce l'Apolomeration Grenobloise

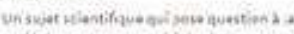

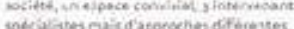
inmotemeit

with ber semente

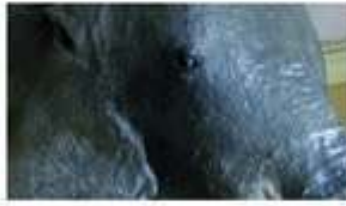

De Memoire dolephant

Depusico ans, Eulate recepante

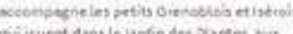

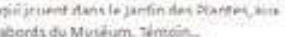

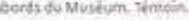

seres neroemmas

(C) Echosciences 


\section{BIBLIOGRAPHIE}

Chicoineau, L. Numérique : vers un nouvel âge de la médiation culturelle des sciences ? La Lettre de l'OCIM, n¹62, 2015, pp. 58-62.

Chicoineau, L. Partager les cultures scientifique, technique et industrielle à l'ère du numérique. Grenoble, 2015, 92 p.

Magro, S. De l'usage des réseaux socio-numériques comme supports d'une médiation culturelle en ligne, La Lettre de l'OCIM, n¹62, 2015, pp. 37-40.

Coville, M. Des réseaux sociaux au FabLab : la culture scientifique et ses publics. Entretien avec Marion Sabourdy, Revue Poli, $\mathrm{n}^{\circ} 8,2014$.

Cordier, S. Culture scientifique et territoires, Culture et Recherche, $\mathrm{n}^{\circ} 132$, automne-hiver 2015-2016.

Fiche ressource de l'atelier « Cartes collaboratives » mené lors des journées 2DaysUp! 2015, par le CNAM Pays-de-la-Loire à Nantes.

Animer des communautés d'intérêt sur les sciences et l'innovation, cahier "communautés" d'Inmédiats, automne 2014

http://inmediats.fr/document/

Bienvenue au XXI siècle - Partager les savoirs et les innovations. Inmédiats, Usbek \& Rica, été 2016 http://inmediats.fr/inmediats/

MOOC Sciences Tag diffusé à l'automne 2016, Inmédiats.

Réseaux sociaux et blogs de sciences et de culture scientifique. $2^{\mathrm{e}}$ Journée d'études du Projet d'animation scientifique ARC 5, Région Rhône Alpes, 30 novembre 2012, Lyon.

\section{NOTES}

1. Dans le cadre d'Inmédiats (http://inmediats.fr/), un programme pensé par six centres de sciences en France : Cap Sciences (Bordeaux), Espace des sciences (Rennes), La Casemate (Grenoble), Science Animation (Toulouse), Relais d'sciences (Caen) et Universcience (Paris)

2. Chicoineau, L. Retour sur l'aventure d'Echosciences Grenoble. echosciences-grenoble.fr, juillet 2012. 3. Le web social fait référence à une vision d'Internet considéré comme un espace de socialisation, un lieu dont l'une des fonctions principales est de faire interagir les utilisateurs entre eux afin dassurer une production continuelle de contenu. Il est associé à différents systèmes sociaux tels que le réseautage social, les blogs ou les wikis (source : Wikipédia).

4. Voir notamment l'expérience du Café des sciences ou de l'Agence Science Presse au Québec.

5. Amateur au sens de "celui qui aime" et non en opposition au professionnel.

6. Sources : outil de statistiques interne au 16/12/2016 et Google Analytics au 25/01/2017.

7. L'agence Probesys pour la première version puis l'agence Sleede et le designer Ugo Retz pour la version actuelle.

8. Un lecteur peut recommander un contenu via un bouton dédié (l'équivalent du "like" de Facebook) et commenter un contenu grâce au service de commentaires centralisés Disqus. 
9. Lire notamment Gauthier, C. Quel rôle pour les muséums dans la production et la diffusion de la connaissance naturaliste à l'époque d'internet? La Lettre de l'OCIM, n¹66, 2016, pp. 5-12.

10. "Application Programming Interface" ou interface de programmation: ensemble normalisé de classes, de méthodes ou de fonctions qui sert de façade par laquelle un logiciel offre des services à d'autres logiciels (source : Wikipédia).

INDEX

Mots-clés : Réseau social, plateforme, web, communauté

\section{AUTEUR}

\section{MARION SABOURDY}

Responsable des nouveaux médias à la Casemate, le Centre de culture scientifique, technique et industrielle de Grenoble

marion.sabourdy@lacasemate.fr 